University of Wollongong

Research Online

Faculty of Engineering - Papers (Archive)

Faculty of Engineering and Information

Sciences

$1-1-2012$

\title{
Microstructure and mechanical properties of IF steel deformed during plane stress local torsion
}

\author{
A H. Shamdani \\ Monash University \\ S Khoddam \\ Monash University \\ P F. Thomson \\ Monash University \\ A Dehghan-Manshadi \\ University of Wollongong, alidm@uow.edu.au
}

Follow this and additional works at: https://ro.uow.edu.au/engpapers

Part of the Engineering Commons

https://ro.uow.edu.au/engpapers/5318

\section{Recommended Citation}

Shamdani, A H.; Khoddam, S; Thomson, P F.; and Dehghan-Manshadi, A: Microstructure and mechanical properties of IF steel deformed during plane stress local torsion 2012, 1582-1587.

https://ro.uow.edu.au/engpapers/5318

Research Online is the open access institutional repository for the University of Wollongong. For further information contact the UOW Library: research-pubs@uow.edu.au 


\title{
Microstructure and Mechanical Properties of IF steel
}

\section{Deformed during Plane Stress Local Torsion}

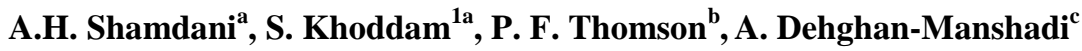 \\ ${ }^{\text {a }}$ Department of Mechanical \& Aerospace Engineering, Monash University, Clayton, Victoria 3800, Australia \\ ${ }^{\mathbf{b}}$ Department of Materials Engineering, Monash University, Clayton, Victoria 3800, Australia \\ ${ }^{\mathbf{c}}$ Faculty of Engineering, University of Wollongong, NSW 2522, Australia
}

\begin{abstract}
A novel localized severe plastic deformation process for reinforcement of fastener holes is presented. This process is capable of inducing large shearing strains confined to an axi-symmetric zone around a hole. The process was applied to thin interstitial free steel samples. In the material around the hole, significant grain refinement was achieved, and a gradient of refinement was observed moving away from the hole. The ultimate and yield strengths were increased by $28 \%$ and $100 \%$ respectively.
\end{abstract}

Keywords: Severe plastic deformation (SPD); Grain refinement; Local torsion; Microstructure; Interstitial free (IF) steel

\section{Introduction}

Mechanical joints are inherently vulnerable to failure because the presence of the joint hole causes a stress concentration in the vicinity of the hole. The need for improvement of material strength around a fastener hole can be satisfied by severe plastic deformation (SPD) to produce ultrafine grains. The ultrafine grained (UFG) alloys produced by SPD processing possess higher strengths than their coarse-grained counterparts as a result of the reduced grain size. However, in some circumstances such as SPD processing of $\mathrm{Al}-\mathrm{Zn}$ and $\mathrm{Al}-\mathrm{Mg}$ alloys the decomposition of supersaturated solid solutions competes with the Hall-Petch effect and leads to a more pronounced softening of the material [1]. Another drawback of SPD processes is that they involve bulk deformation and large energy consumption [2]. It is therefore desirable to enhance the global behaviour of the material by limiting improvement of the material property by SPD to the location at which it is needed. Localized severe plastic deformation (LSPD) techniques, such as forward spiral extrusion [3] and friction stir processing [4], involve lower energy

\footnotetext{
${ }^{1}$ Corresponding author Tel.: +61-3-99058656

E-mail address: shahin.khoddam@ monash.edu

Address: Room 118, Building 31, Mechanical \& Aerospace Engineering Department,

Monash University, Clayton, Victoria 3800, Australia
} 
consumption. They modify the properties of materials locally and create a gradient of grain refinement, resulting in significant improvement in the mechanical properties of the processed samples. However, these techniques cannot be used for strengthening the material around fastener holes, and thus a method for improving the strength of material around the hole is needed.

To reinforce the mechanical properties of material around a hole, the plane stress local torsion (PSLT) process, which involves a plane stress axi-symmetric torsional loading, is introduced. The PSLT takes advantage of large shearing strains induced around the intended hole position, through torsional deformation [5]. As a result, the material flows plastically within a thin annular zone around the fastener hole $(\mathrm{AZFH})$. Because of the limited penetration of the flow localization zone into the material, a major proportion of deformation energy is consumed within the AZFH. The PSLT therefore consumes much less energy than do bulk grain refinement techniques.

\section{Principles of the PSLT}

In the case of a thick-walled cylinder subject to a shearing load on its inner surface, the plastic shear strain, $\varepsilon_{r \theta}$, in the zone surrounding the hole can be calculated using a tangential displacement correlation given in [6]:

$$
\varepsilon_{r \theta}=\frac{M(1+v)}{2 \pi r^{2} E}-\frac{2 \log (\rho / r)}{\eta}
$$

where $r$ is the distance from the centre of the hole, $E$ is Young's modulus, $v$ is Poisson's ratio, $\eta$ is a constant assuming the material hardens isotropically, $\rho=\sqrt{M / 2 \pi \sigma_{y s}}$ is the radius of elastic-plastic interface), $M$ is the applied torque per unit thickness, and $\sigma_{y s}$ is the yield stress in shear. According to Eq. (1) above, the shear strain is the sum of two terms, the first inversely proportional to $r^{2}$, the second inversely proportional to $\log (r)$. Which term dominates depends on geometrical and material parameters, but it can be expected that the first dominates at sufficiently small $r$ (for small holes), whereas the second dominates at greater radii. The logarithmic distribution of strain and its inverse proportionality to $r$, suggests gradation in the radial direction, producing a zone of intense grain refinement and enhanced mechanical properties, declining to a more diffuse change, which confers an advantage of PSLT over other SPD methods.

The process of PSLT is implemented using a rig developed in-house (Fig. 1(a)). In the PSLT process, a thin plate specimen (Fig. 1(b)) is treated locally, before hole drilling. A built-in mandrel, which is a part of the specimen, applies the torque at the intended hole position. A socket head which is held by a three- 
jaw chuck (Fig. 1(a)) transfers the torque through the mandrel to the specimen. The confining die, which holds the specimen, is positioned in a fixture plate and keeps the specimen stationary at its external boundary during the process (Fig. 1(b)).

The PSLT was applied to deform Ti-IF (interstitial free) steel specimens having a thickness of $1 \mathrm{~mm}$ and an intended hole diameter of $10 \mathrm{~mm}$ in the centre. The required torque was applied with the input power of $0.108 \mathrm{~kW}$ and rotation rate of $0.0087 \mathrm{rad} / \mathrm{s}$. The torque and angle of rotation was monitored during the test and continued until it dropped sharply (an indication of macro-crack initiation). The plastically deformed zone can serve as a reinforced fastener hole after machining the mandrel and drilling a $10 \mathrm{~mm}$ hole concentric with the AZFH. As depicted in Fig. 2, a torque of $49 \mathrm{Nm}$ was required to twist the material within the AZFH through 110 degrees, which corresponds to a strain of $\varepsilon_{r \theta}=3$ at the edge of the intended hole.

To characterize the extension of the plastically deformed zone visually, the surface of the sample was gridded and the grids were measured after the sample was deformed to failure. The initially-radial grids on the specimen were distorted after deformation within a limited distance from the edge of the hole where the polished surface of the specimen became irregular.

\section{Results and discussion}

The micro shear punch (MSP) test was used to evaluate the improvement in mechanical strength of the deformed zone. In the MSP test, the ultimate strength of the material is estimated from the maximum point on the force-displacement curve [7]. MSP tests were performed within plastically deformed and undeformed zones of the specimen at a speed of $0.004 \mathrm{~mm} / \mathrm{s}$. The resultant force-displacement curves, as illustrated in Fig. 3, were recorded using dedicated data acquisition software.

The difference between the peak load for the deformed region $(1548 \mathrm{~N})$ and that for the un-deformed region $(1214 \mathrm{~N})$ indicates approximately an improvement of $28 \%$ in the ultimate strength within the AZFH. Due to the large and nonlinear gradient of deformation and grain refinement within the AZFH, and a relatively large footprint of $1.5 \mathrm{~mm}$ in diameter of the micro shear punch, the $28 \%$ improvement should be interpreted carefully. Given that only a small portion of the punch boundary would be in the maximum grain refinement zone, it is concluded that the improvement in the zone would be significantly higher than $28 \%$. In both curves, the gradual reduction in the shear force after the peak point indicates ductile fracture of the specimen. Fracture behaviour of IF steel could be affected by the PSLT processing as a result of reduction in the grain sizes similar to bcc metals under high pressure torsion [8]. 
In addition to the MSP test, microhardness tests were used to investigate the influence of the PSLT process on the specimen. There is a linear correlation between microhardness and yield strength over data in a limited range [9]. The microhardness tests were performed at five points and the measured hardness (HV) values as a function of distance from the hole edge, $r-r_{i}(\mathrm{~mm})$, are reported in Table 1 . By extrapolating and comparing hardness values at $r-r_{i}=0.05$ and $8 \mathrm{~mm}$, an increase of nearly $100 \%$ in the yield strength of the material was found. The result of the microhardness test showed a radial increase in mechanical strength, as suggested by Eq. 1.

Moreover, the microstructure of the specimens tested was examined by optical microscopy (OM). Samples were cut and prepared at the $z=0$ plane (see Fig. 1(b)). As shown in Fig. 4(a), the grains outside the deformed zone were equi-axed. In Fig. 4(b), a narrow band of quite severely-refined grains about $300 \mu \mathrm{m}$ wide near the edge of the hole, in a pure shearing deformation mode, can be distinguished from the rest of the material. In this heavily deformed region, where the shear strain is substantially raised, the distribution of shear strain is heterogeneous which is typical for most SPD processes involving large shearing deformations [10]. The heterogeneity is reduced as moving away from the edge of the hole in the radial direction. The OM results also suggest that the shear deformation is more efficiently enforced on the material closer to the edge of the intended hole. Fig. 4(b) shows the gradient in the accumulated strain and grain size in the sample, which correlates with the gradient in mechanical properties measured by MSP tests and microhardness.

Flow localization through the formation of shear bands, where the energy of deformation is accumulated, can be observed in the areas of prevailing microstructural refinement as shown in Fig. 4(c). Fig. 4(c) shows the shape and orientation of the grains within the plastically deformed zone. The heavy deformation of material and very large strains induce a fine grain structure very close to the edge of the hole. Grains are elongated in the tangential direction, $\theta$, and thinned in the transverse direction, $r$. The microstructure of the material very close to the edge of the hole appears fibrous and complex flow patterns could be present due to the local shear deformation gradients [11]. The gradient of the grain refinement in the $z$ direction was negligible due to the small thickness of the specimen.

Grain sizes expressed in terms of the width $(W)$ in the $\theta$ direction and height $(H)$ in the $r$ direction, and the grain aspect ratio, $A R=W / H$, are shown in Table 2 as a function of $r-r_{i}(m m)$. The values shown in the table are the average of five measurements at each distance from the edge of the hole. As shown in Table 2, the aspect ratio of grains increases as the distance from the edge of the hole decreases. 
The large aspect ratio of grains very close to the hole correlates with the elongation of grains in the tangential direction. Extrapolating the data given in Table 2 indicates that grains located at the edge of the hole are expected to have an aspect ratio of approximately 15.

The PSLT process showed a strong grain refinement in the heavily deformed area, close to the edge of the hole. The grain refinement reduced rapidly toward the external edge of the sample up to $4 \mathrm{~mm}$ from the hole edge. The EBSD maps (Figs 5(a) and (b)) show a typical example of microstructure obtained in the heavily deformed and less deformed areas of the specimen respectively. This microstructure is a composite structure of ultrafine grains of $0.2-0.5 \mu \mathrm{m}$ together with larger and elongated grains. The presence of such a heterogeneous grain refinement is expected in many deformation processes and depends somewhat on the grains crystallographic orientation (prior to deformation). However, a considerable improvement in the mechanical properties is expected accompanying grain refinement by development of sub-micron grains and/or subgrains as shown in the EBSD map of material in the deformed zone (Fig. 6).

As shown in Fig. 6, the severe plastic deformation during the PSLT process has introduced high angle grain boundaries (HAGB), misorientation $\geq 15$ degrees, as well as the low angle grain boundaries (LAGB), misorientation $<2$ degrees, in the microstructure, which are distinguished by black and red lines respectively. The ultrafine and elongated grains with the HAGBs in the microstructure impede the motion of dislocations and consequently enhance the strength. Other deformation mechanisms such as grain boundary sliding and grain rotation may also be facilitated by the HAGBs [12]. Fig. 6 clearly shows the presence of a relatively well defined and sub-micron size low-angle grain boundary network within the elongated grains. The presence of such a low angle grain boundaries network (sub-boundaries) could also improve the mechanical properties of the material.

As shown in EBSD maps, the presence of the ultrafine grain structure within the AZFH facilitates the strain hardening of the material and causes a delay in occurrence of the plastic instability which leads to continuous flow of the material. Moreover, the HAGBs of the ultra-fine grains generated by the PSLT method, as a SPD process, are usually in a non-equilibrium state [13]. These grain boundaries provide a higher density of dislocations for slip and slide of grains compared to LAGBs. Movement of these dislocations and the dislocations piled up close to the grain boundaries facilitate the grain boundary sliding and the grain rotation resulting in an increase in the formability [13-15]. In the areas of high shear strain the availability of a bimodal microstructure (a mixture of grains with varying size and properties) 
including ultra-fine grains along with elongated ones can be observed [16]. The strengthening from the ultrafine grain structure, with a grain size of less than $1 \mu \mathrm{m}$, together with increased formability provided by dislocation activity in larger grains conduces to an optimal state of high strength and acceptable formability within the AZFH.

\section{Conclusions}

In conclusion, a new process, plane stress local torsion (PSLT), has been proposed which can induce large plastic shearing strains within the material adjacent to a hole. An overall increase of approximately $100 \%$ in yield strength and $28 \%$ in ultimate strength of the material in the flow-localized zone over the punched zone has been demonstrated. Investigation of the microstructure of the material and its microhardness confirmed the gradient of grain refinement in the radial direction. The microstructure also showed highly elongated and refined grains in the flow localization zone. The ultrafine grain boundaries, as an effective source of dislocations, together with a bimodal distribution of grain sizes, provide possible means to increase strength and formability in the ultrafine grained Ti-IF steel processed by PSLT. A major advantage of PSLT is its ability to concentrate grain refinement around an intended hole position, which makes PSLT a more flexible and versatile process that requires less energy consumption. A potential application for PSLT is local reinforcement of fastener holes which are extensively used in mechanical structures.

\section{Acknowledgement}

The authors acknowledge the financial support of the Australian Cooperative Research Centre for Advanced Automotive Technology (AutoCRC) in this project.

\section{References}

[1] Mazilkin AA, Straumal BB, Rabkin E, Baretzky B, Enders S, Protasova SG, Kogtenkova OA, Valiev RZ (2006) Acta Mater. 54:3933-3939.

[2] Zhilyaev AP, Langdon TG (2008) Prog. Mater. Sci. 53:893-979.

[3] Khoddam S, Farhoumand A, Hodgson PD (2011) Mat. Sci. Eng. A-Struct. 528:1023-1029.

[4] Kwon YJ, Saito N, Shigematsu I (2002) J. Mater. Sci. 21:1473-1476.

[5] Shamdani AH, Khoddam S (2011) Arch. Appl. Mech. doi: 10.1007/s00419-011-0566-4.

[6] Nordgren RP, Naghdi PM (1963) Int. J. Eng. Sci. 1:33-70.

[7] Lucas GE (1983) J. Nucl. Mater. 117:327-339. 
[8] Hohenwarter A, Kammerhofer C, Pippan R (2010) J. Mater. Sci. 45:4805-4812.

[9] Cahoon JR, Broughton WH, Kutzak AR (1971) Metall. Mater. Trans. B 2:1979-1983.

[10]Zrnik J, Pippan R, Scheriau S, Kraus L, Fujda M (2010) J. Mater. Sci. 45:4822-4826.

[11]Cao Y, Kawasaki M, Wang YB, Alhajeri SN, Liao XZ, Zheng WL, Ringer SP, Zhu YT, Langdon TG (2010) J. Mater. Sci. 45: 4545-4553.

[12] Valiev RZ, Alexandrov IV, Zhu YT, Lowe TC (2002) J. Mater. Res. 17:5-8.

[13] Valiev RZ, Islamgaliev RK, Alexandrov IV (2000) Prog. Mater. Sci. 45:103-189.

[14] Peeters B, Kalidindi SR, Van Houtte P, Aernoudt E (2000) Acta mater. 48:2123-2133.

[15] Hasnaoui A, Van Swygenhoven H, Derlet PM (2002) Acta Mater. 50:3927-3939.

[16] Fatemi-Varzaneh SM, Zarei-Hanzaki A, Izadi S (2011) J. Mater. Sci. 46:1937-1944. 


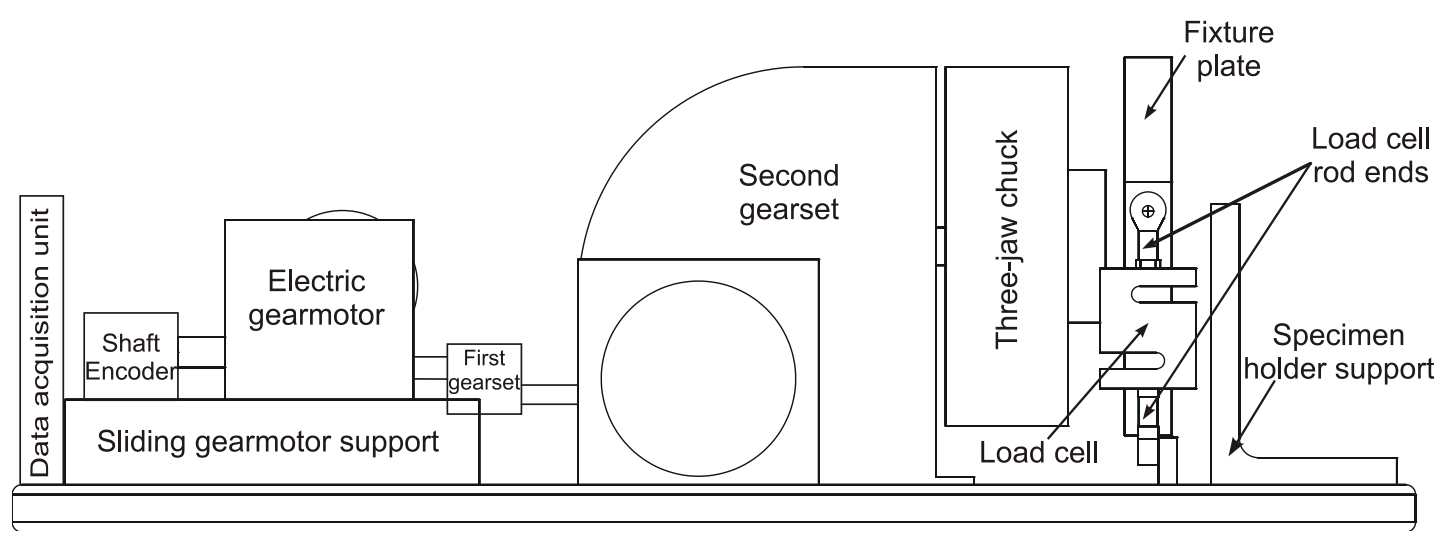

(a)

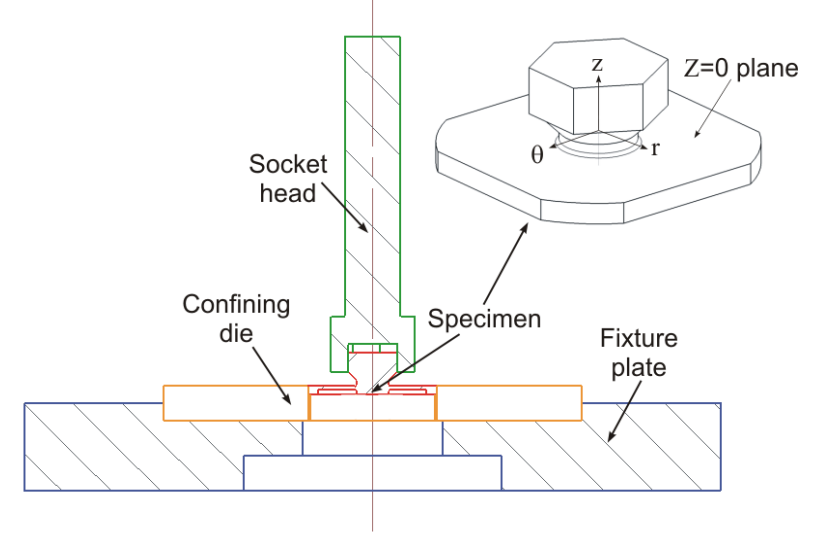

(b)

Figure 1. Schematic view of the experimental PSLT setup, (a) PSLT apparatus and (b) specimen fixture assembly

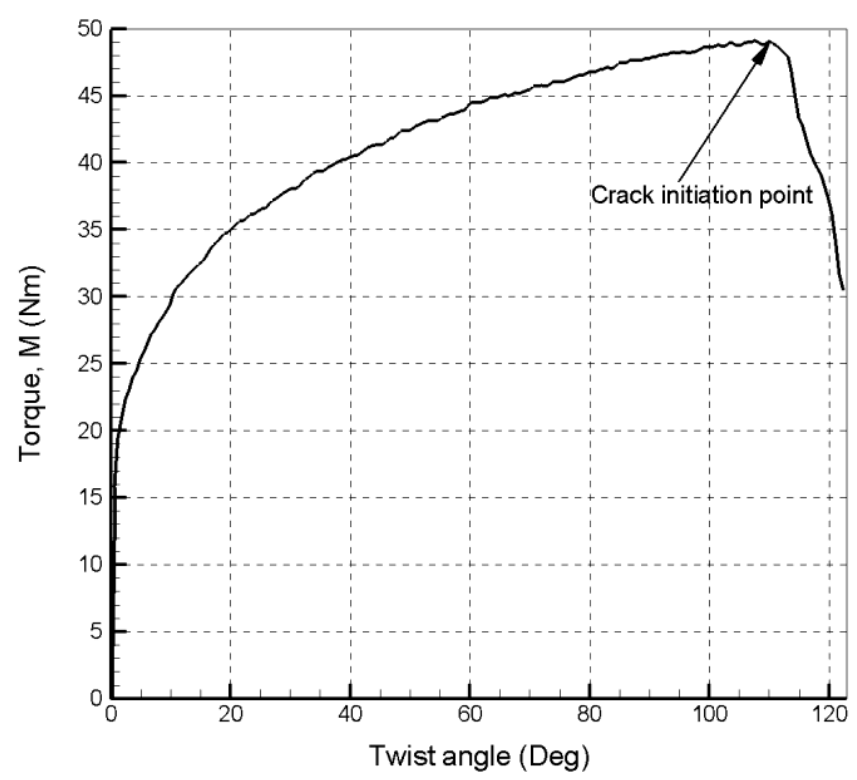

Figure 2. Torque-twist curve of IF steel with intended hole diameter of $10 \mathrm{~mm}$ 


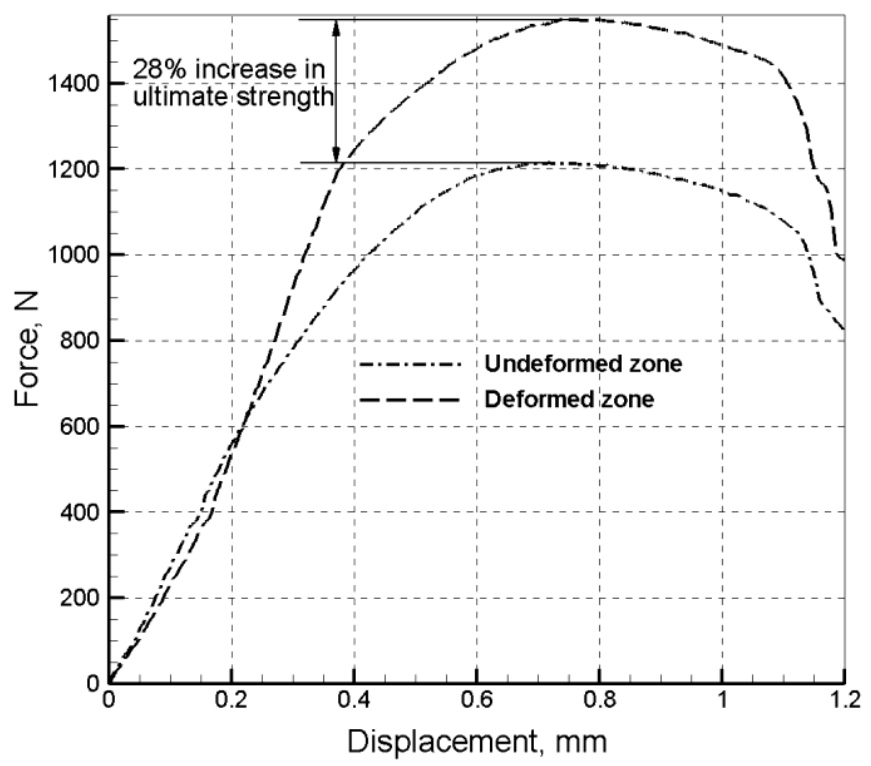

Figure 3. Shear force-displacement of IF steel for deformed and undeformed regions

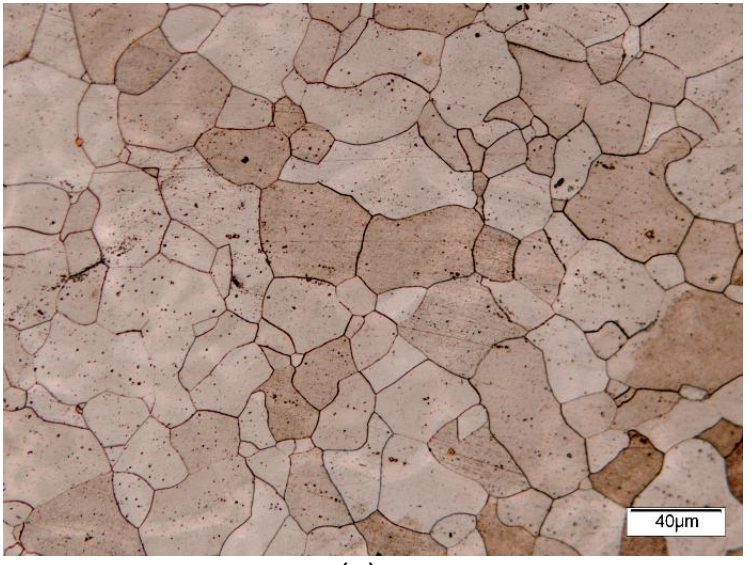

(a)

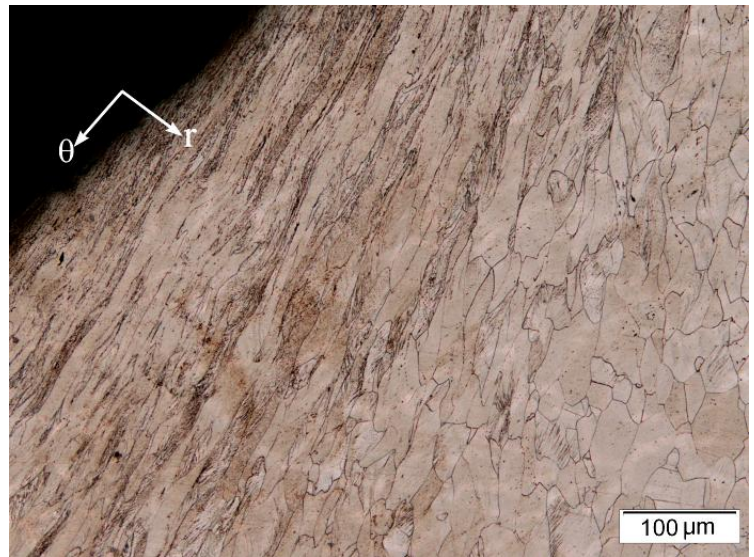

(b)

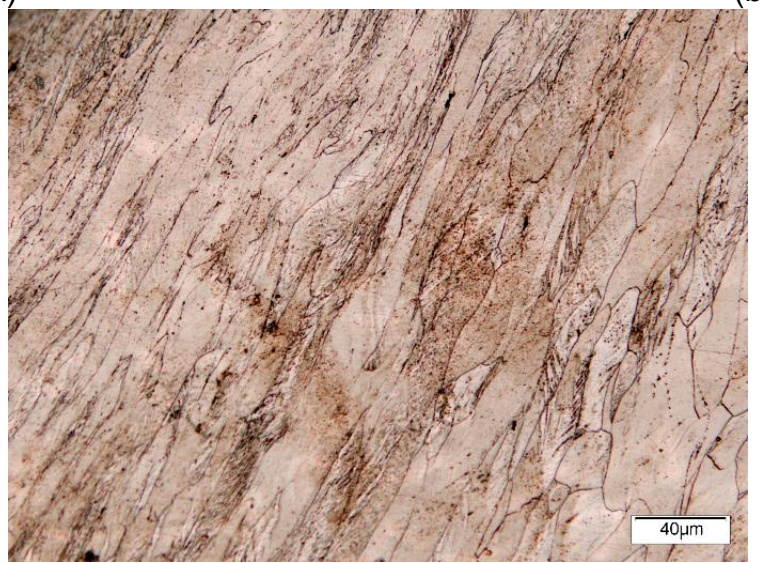

(c)

Figure 4. Metallographic pictures of Ti-IF steel specimen processed by PSLT. Photos (a-c) are in the $r-\theta$ plane at $z=0$ 


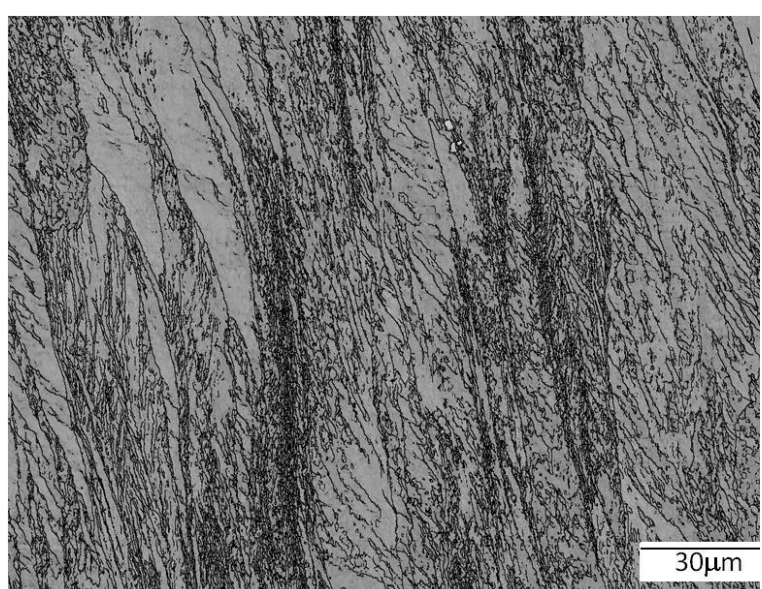

(a)

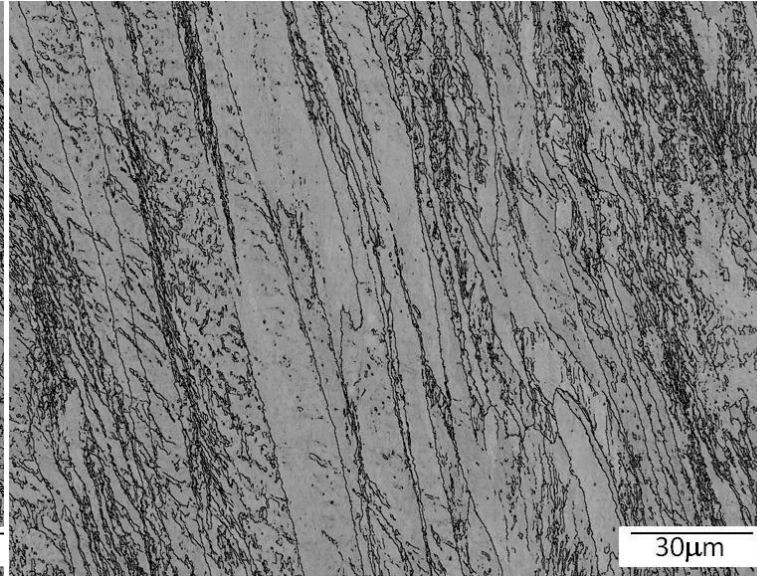

(b)

Figure 5. EBSD maps from the heavily deformed zone of the specimen (a) very close to the edge of the hole, and (b) farther from edge of the hole

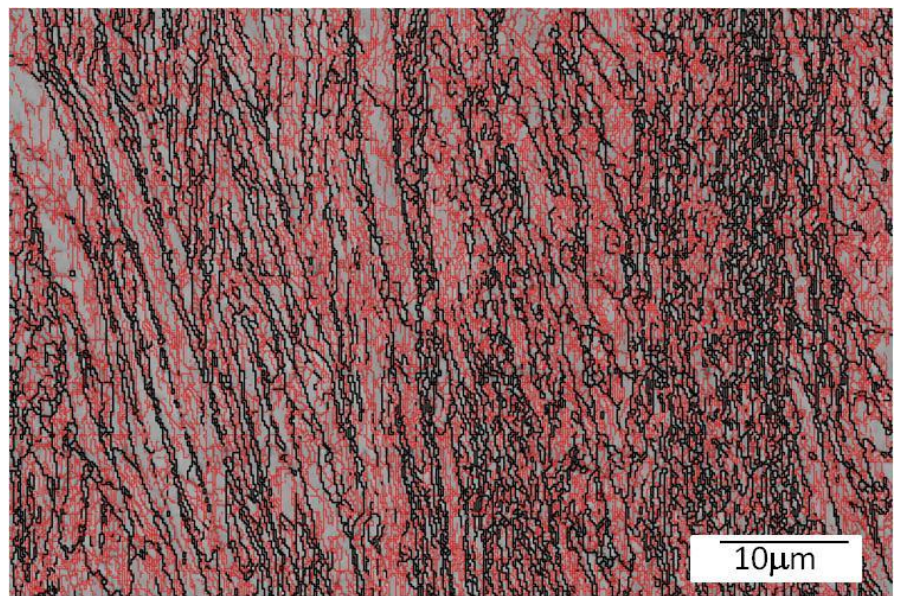

Figure 6. EBSD map of the heavily deformed zone showing high angle and low angle grain boundaries (red line: low angle grain boundaries $\left(<2^{\circ}\right)$, black line: high angle grain boundaries $\left(>15^{\circ}\right)$ 